Trauma Berufskrankh 2009 · 11[Suppl 1]:60-64 DOI 10.1007/s10039-008-1411-z

Online publiziert: 5 . September 2008

c) Springer Medizin Verlag 2008

\author{
M. Faschingbauer $\cdot$ K. Seide \\ Abteilung für Unfall- und Wiederherstellungschirurgie, \\ BG-Unfallkrankenhaus, Hamburg
}

\section{Das intelligente Implantat}

\section{Methode}

folgt routinemäßig durch Röntgenaufnahmen. Um indirekt von der radiologischen knöchernen Durchbauung auf die Steigerung der Belastung der Fraktur zu schließen, bedarf es großer Erfahrung des behandelnden Chirurgen. In Fällen mit Problemen in der Frakturheilung lassen sich oft durch nativ-radiologische Untersuchungen keine entscheidenden Aussagen tätigen, weshalb dann Zusatzuntersuchungen - wie CT oder Schichtaufnahmen - mit z. T. erhöhter Strahlbelastung nötig sind.

Um eine objektivere Beurteilung der Steifigkeit einer Osteosynthese zu erlangen, wurden bereits früher Osteosynthesesysteme entwickelt, welche Last, Biegungen oder Dehnungen messen können. Diese wurden bereits erfolgreich eingesetzt, und zwar vorwiegend bei Fixateurexterne-Systemen $[1,2,3,6]$, wohingegen bei internen Systemen bisher wenig Erfahrungen vorliegen $[4,5]$.

Über das Forschungsprojekt INOS (Intelligente Osteosynthese), gefördert durch den Hauptverband der gewerblichen Berufsgenossenschaften (HVGB), wurde eine Entwicklung von Fixateur-interneSystemen als „Intelligente Implantate“ im BG-Unfallkrankenhaus Hamburg mit der Technischen Universität Hamburg-Harburg durchgeführt. Durch das Aufbringen einer modernen Mikroelektronik auf eine Platte ist es möglich, mittels RFIDTechnologie (Radiofrequenzidentifikationstechnologie) telemetrisch die Belastung des Implantats zu messen. Aufgrund der kleinen Dimensionierung eines einfachen und relativ kostengünstigen Sensorsystems kann dieses in interne Plattensysteme integriert werden.
Es wurde eine miniaturisierte telemetrische Messeinheit entwickelt, auf handelsübliche winkelstabile Fixateur-interne-Systeme appliziert und biokompatibel verkapselt (• Abb. 1). Diese Transponder werden „passiv“ durch ein Lesegerät mit Energie versorgt (induktive Kopplung), sodass eine Batterie nicht erforderlich ist. Als Sensoren dienen Dehnungsmessstreifen.

Für die klinische Anwendung wurde eine extrakorporale portable Leseeinheit entwickelt, welche telemetrisch die Daten des Miniaturtransponders aufnehmen kann [7]. Sie werden von dieser Einheit in ein Computermesssytem übertragen, mit welchem gleichzeitig äußere Lasten und ein simultan durchgeführtes Elektromyogramm registriert werden können.

Zunächst wurden in Versuchen an Kunststoffmodellen verschiedener Fraktursituationen die in vivo zu erwartenden Messwerte simuliert und die Funktionalität des Gesamtsystems nachgewiesen.

Anschließend wurde das System im Tierversuch geprüft. Instrumentierte winkelstabile Fixateur-interne-Systeme $\left(\right.$ TIFIX $\left.^{\oplus}\right)$ in der Dimensionierung für den menschlichen Unterschenkel wurden bei 6 Schafen an der Tibia montiert und eine quere Osteotomie durchgeführt. Nach 12 Wochen Implantationsdauer wurde histologisch die Verträglichkeit nachgewiesen. Parallel dazu erfolgte das Monitoring der knöchernen Durchbauung durch regelmäßige Messungen der Implantatbelastung unter standardisierten äußeren Lasten sowie Röntgenaufnahmen nach 3, 6 und 12 Wochen. Zusätzlich wurde eine spezielle Leseeinheit mit BluetoothSchnittstelle entwickelt, welche als „Rucksacksystem“ (- Abb. 2) kontinuierliche Messungen über Funkverbindung bei frei laufendem Tier ermöglichte.

Danach erfolgten die ersten klinischen Anwendungen an bisher 5 Patienten.

\section{Ergebnisse}

\section{Tierversuche}

Sie zeigten, dass der Frakturheilungsverlauf mit dem System kontrolliert und quantifiziert werden kann. Dazu wurde eine relative Steifigkeit als Verhältnis der auf die Extremität aufgebrachten Kraft und der im Implantat gemessenen Biegelast bestimmt. Aufgrund der Verschiebung des Kraftflusses von der Platte auf den zunehmend steiferen Knochen nimmt diese relative Steifigkeit im Verlauf der regulären Knochenheilung zu. Es zeigten sich darüber hinaus ein biomechanisch interessanter Einfluss muskulärer Regelprozesse, so fand sich z. B. bereits vor dem Beginn einer Laufbewegung eine Anspannung, welche nach dem Stillstand nach einigen Sekunden langsam abklang.

Im Rahmen der Verträglichkeitsprüfung durchgeführte Vergleiche zwischen Gewebe am Plattenende (entspricht herkömmlichem Implantat) und Plattenmitte

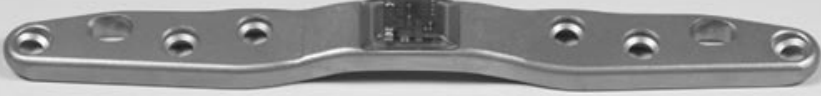

Abb. $1 \Delta$ Mit Miniaturtelemetrie instrumentierter Femurfixateur interne 


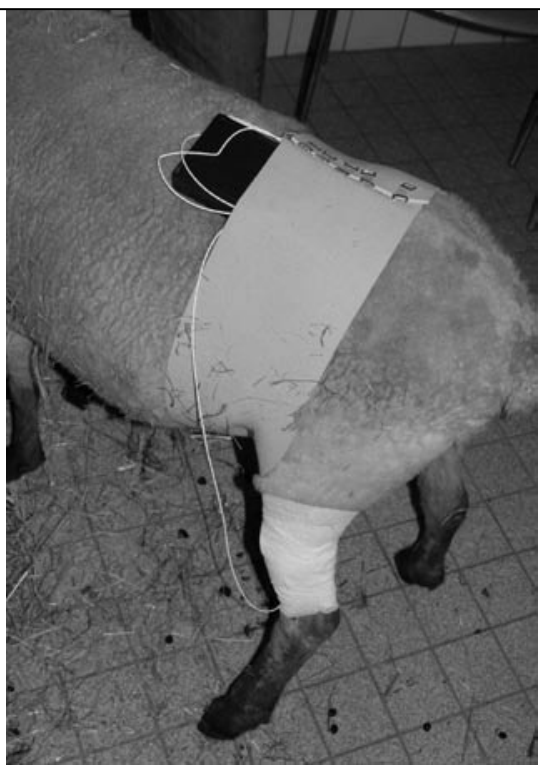

Abb. $2 \Delta$ Entwickelte Leseeinheit mit Bluetooth-Funkübertragung, als, Rucksacksystem" Möglichkeit kontinuierlicher Messungen über Funkverbindung bei frei laufendem Tier

(mit verkapseltem Sensorsystem) zeigten sich keine Unterschiede der Zellreaktion oder andere Auffälligkeiten.

\section{Klinik}

Die klinische Anwendung erfolgte bei 5 Patienten mit mehrfach voroperierten Pseudarthrosen sowohl am distalen und proximalen Oberschenkel als auch in Schaftbereich (• Abb. 3). Diese Problemfälle wurden gewählt, um einerseits mögliche Ursachen der Heilungsprobleme zu erkennen und andererseits das Risiko einer weiteren Fehlheilung durch optimierte Laststeuerung zu minimieren.

Bei den ausgesuchten Patienten waren unterschiedliche Implantate bei den Primärversorgungen eingesetzt worden. Diese reichten vom ante- und retrograden Nagel über LCDC-Platten bis zur winkelstabilen Platte.

In allen Fällen erfolgte die Versorgung mit einem instrumentierten Oberschenkel-TIFIX ${ }^{\circledast}$ (multidirektional winkelstabiles Implantat auf Grundlage einer Wellenplatte, $\bullet$ Abb. 1). Zusätzlich wurden eine Spongiosaplastik durchgeführt und eine PMMA-Kette zur Infektionsprophylaxe eingelegt, da sich in einer hausinternen Studie in über der Hälfte der Fälle von Oberschenkelpseudarthrosen ein LowGrade-Infekt hatte nachweisen lassen. Die Ergebnisse der postoperativen Behand-
Trauma Berufskrankh 2009 - 11[Suppl 1]:60-64 DOI 10.1007/s10039-008-1411-z

(C) Springer Medizin Verlag 2008

\section{Faschingbauer $\cdot$ K. Seide Das intelligente Implantat}

\section{Zusammenfassung}

Vorgestellt wird ein im BG-Unfallkrankenhaus Hamburg zusammen mit der Technischen Universität Hamburg-Harburg neu entwickeltes als "Intelligentes Implantat" bezeichnetes Fixateur-interne-System. Durch das Aufbringen einer modernen Mikroelektronik auf eine Platte ist es möglich, deren Belastung telemetrisch zu messen. Aufgrund der kleinen Dimensionierung des Sensorsystems kann dieses in interne Plattensysteme integriert werden. Das System wurde zunächst in Kunststoffmodellen, anschließend im Tierversuch geprüft. Danach erfolgten die ersten klinischen Anwendungen an Patienten. Laut Tierversuch kann der Frakturheilungsverlauf mit dem System kontrolliert und quantifiziert werden. Die ersten Ergebnisse bei den Patienten mit Femurpseudarthrosen zeigten ei-

\section{The intelligent implant}

\section{Abstract}

This article presents an "intelligent" internal fixator system developed by the BG-Unfallkrankenhaus in Hamburg in cooperation with the Technical University Hamburg-Harburg. By application of modern microelectronics to a plate, telemetric load measuring is possible. Due to its small size, the sensor system can be integrated in internal plate systems. The system was initially tested in vitro on plastic bones and then tested in animal studies. After this the first clinical applications on patients were carried out. According to results from animal studies, the system enables fracture healing progression to be monitored and quantified. Initial results in patients with femoral non-unions demonstrate ne gute Korrelation zwischen der knöchernen Heilung und dem Verlauf der empfangenen Signale. Der routinemäßige Einsatz dieser Systeme würde eine telemetrisch kontrollierte Nachbehandlung von Osteosynthesen mit optimaler Ausnutzung der Belastungsmöglichkeit der Extremität erlauben. Zudem könnten Probleme in der Frakturheilung frühzeitig erkannt und diesen entsprechend begegnet werden. „Intelligente" Implantate werden u. E. für die Osteosynthese der Zukunft wichtige Werkzeuge darstellen.

\section{Schlüsselwörter}

Fixateur-interne-System · „Intelligentes Implantat" $\cdot$ Telemetrische Messung . Tierversuch · Pseudarthrosenbehandlung good correlation between bone healing and the progression of the signals received. Routine use of this system would enable telemetrically monitored aftercare of osteosyntheses with optimal utilization of load bearing in the extremities. In addition, fracture healing problems could be identified and dealt with promptly. In our opinion, "intelligent" implants will represent an important tool for osteosynthesis of the future.

\section{Keywords}

Internal fixator system ."Intelligent implant" . Telemetric measuring · Animal studies . Non-union treatment 


\section{Was gibt es Neues: Innovative Implantate}

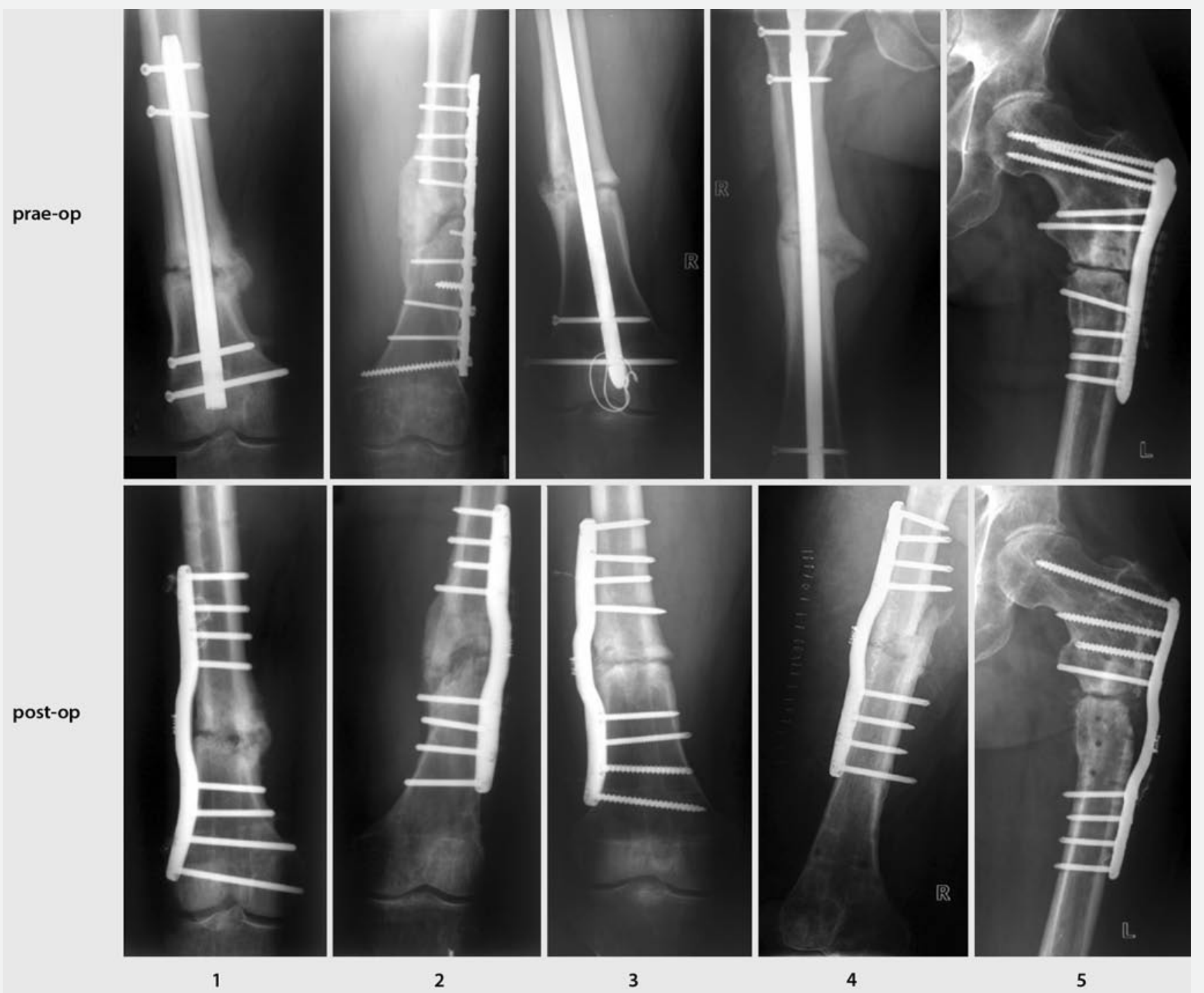

Abb. $3 \Delta$ Röntgenbilder der 5 Patienten $(1,2,3,4,5)$ prä- und postoperativ

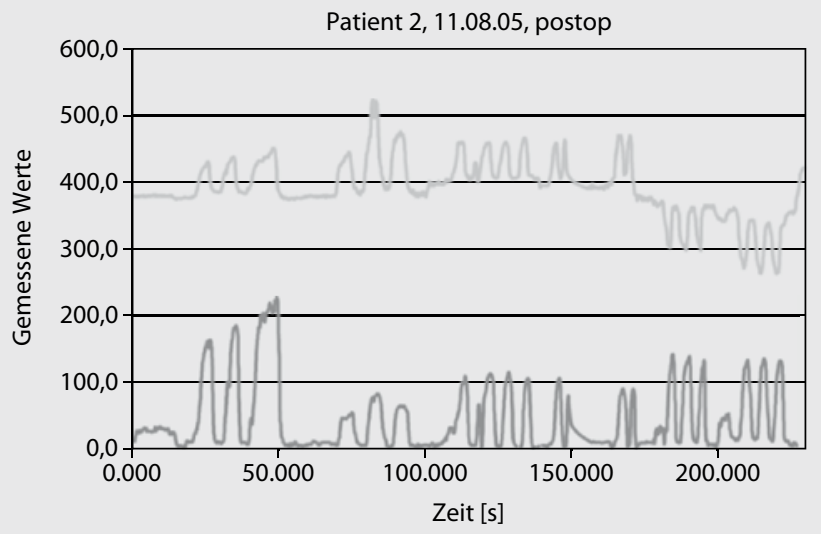

Abb. $4<$ Beispiel einer Messung, telemetrisch gemessene Fixateurbiegebelastung (oben) und äußere Kraft (unten) unter zyklischer axialer (0-50 S), Varus(60-180 S) und Valgusbelastung (180-250 S)
Abb. 5 Fallbeispiel, a röntgenologischer Ausgangsbefund (Pseudarthrose, liegender Femurnagel) und Röntgenbilder nach Reosteosynthese mit intelligentem Implantat, b,c Elastizität der Osteosynthese im Heilungsverlauf unter $\mathbf{b}$ axialer Belastung, $\mathbf{c}$ Varusbelastung, $\mathbf{d}-\mathbf{f}$ postoperative Röntgenbefunde nach $\mathbf{d}$ 32, e 62, f 107 Tagen, $\mathbf{g , h}$ Durchführung kontinuierlicher Messungen der Last im Fixateur interne während krankengymnastischer Übungen (g) und unter axialer Belastung der unteren Extremität (h), Telemetrieantenne der Leseeinheit durch Verband fixiert 

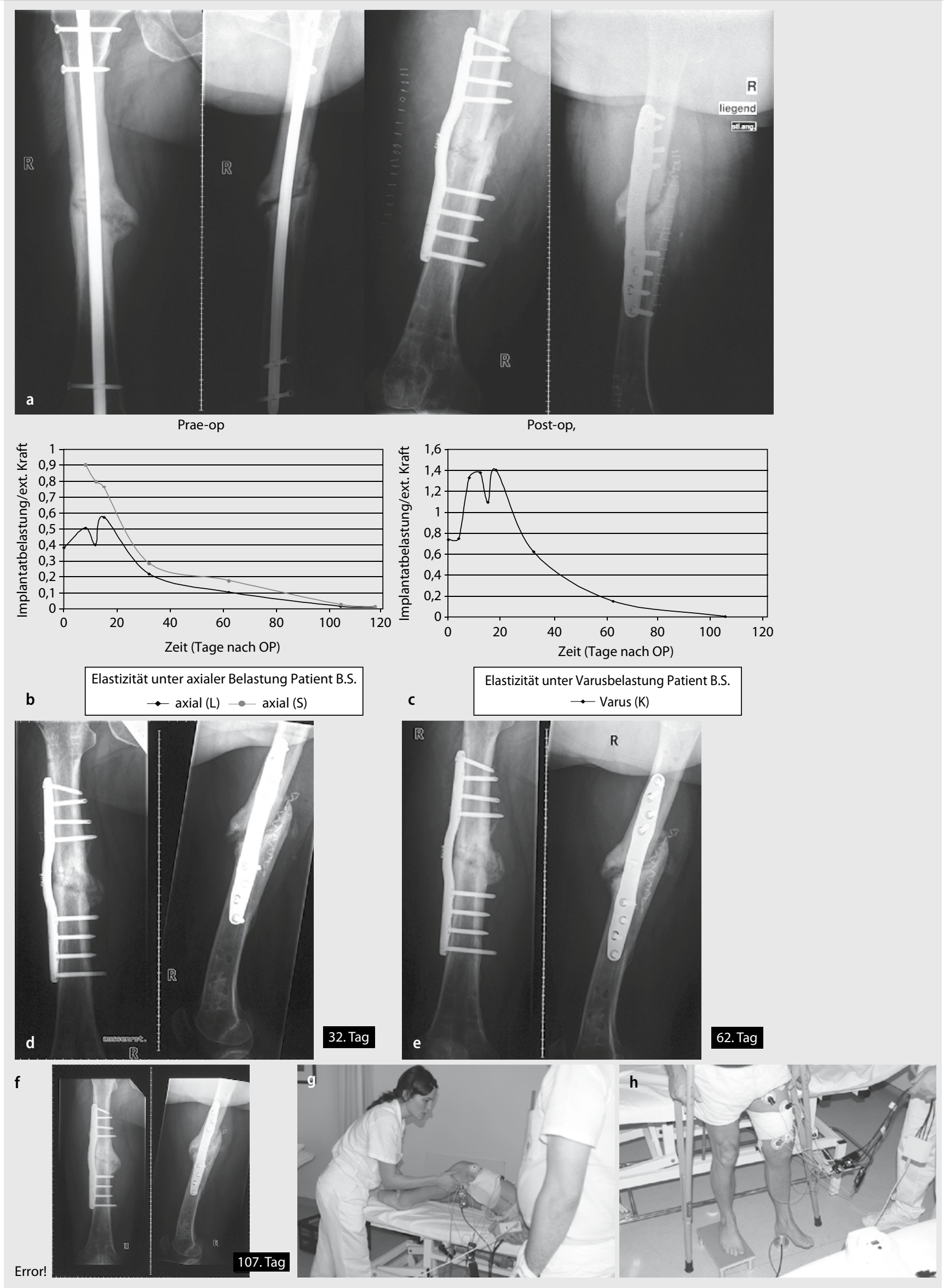
lungen zeigten, dass die telemetrisch ermittelten Plattenbelastungen gut mit den auf die Extremität applizierten zyklischen Lasten korrelierten ( $\bullet$ Abb. 4).

Die auf die äußeren Lasten bezogene Plattenbelastung verringerte sich im Verlauf der Frakturheilung, was exemplarisch am Verlauf eines Patienten dargestellt wird (• Abb. 5).

Kasuistik. Es handelte sich um einen 46jährigen Patienten mit einer vor $17 \mathrm{Mo}$ naten mit antegradem Nagel versorgten Oberschenkelfraktur. 11 Monate nach dem Unfall wurde aufgrund einer Pseudarthrose eine Reosteosynthese mit Marknagel und Spongiosaplastik vorgenommen. Nach weiteren 5 Monaten bestanden keine Zeichen knöcherner Heilung. Aus diesem Grund erfolgten:

- Pseudarthrosenrevision,

- autologe Spongiosaplastik,

- PMMA-Ketten-Einlage und

- Verfahrenswechsel durch Stabilisie-

rung mit dem winkelstabilem intelli-

gentem Implantat ( $\bullet$ Abb. 5a).

Nach 32 Tagen fanden sich messtechnisch ein Rückgang der Elastizität um etwa $40 \%$ ( Abb. 5b,c) und radiologisch keine wesentliche knöcherne Reaktion (- Abb. 5d). Bei der nächsten Messung nach 62 Tagen zeigte sich ein Rückgang der Elastizität auf etwa 10\% (• Abb. 5b,c). Die Röntgenkontrolle war eher ernüchternd, sie ergab keine zunehmende knöcherne Konsolidierung (• Abb.5e). Aufgrund der Messungen wurde von uns Vollbelastung erlaubt. Nach 107 Tagen zeigte sich auch radiologisch eine knöcherne Durchbauung (• Abb. 5f). Messwertetechnisch war zu diesem Zeitpunkt die Elastizität erwartungsgemäß nahezu o (- Abb. 5b,c).

Besonders interessante Ergebnisse fanden sich unter willkürlicher Muskelanspannung sowie bei kontinuierlicher Aufzeichnung der telemetrisch gemessenen Plattenlast während krankengymnastischer Übungen (- Abb.5g,h). Um eine Vergleichbarkeit der durchgeführten Messungen zu erzielen, wurden die Messwerte auf diejenige Last standardisiert (100\%), welche sich bei axialer Last unter $10 \mathrm{~kg}$ zeigte. Diese Last war dem Patienten durch den Operateur als Teilbe- lastung erlaubt. Bei maximaler Anspannung der Oberschenkelmuskulatur wurden Werte bis 500\% registriert. Das Anheben des Beins in Rückenlage ergab bis zu 240\%. Kurzfristige Spitzen (bis 150\%) zeigten sich beim Ablegen des Beins am Schluss einer durch die Physiotherapeutin geführten Bewegung. Übungen mit einer Torsionskomponente ergaben ebenfalls hohe Plattenbelastungen bis $320 \%$. Gab man dem Patienten die Anweisung, das Bein mit voller Muskelanspannung im Oberschenkel zu stabilisieren und dann die Ferse zunehmend zu belasten (bis maximal $30 \mathrm{~kg}$ ), führte die Muskelanspannung zunächst zu einer Implantatbelastung, welche dann beim Belasten der Ferse in ihrem Wert nahezu konstant blieb. Hier ist ein Regelkreis zu postulieren, welcher die zusätzlich auftretende Belastung durch Entspannung der Muskulatur zu kompensieren trachtet.

\section{Diskussion}

Die routinemäßige Anwendung moderner Mikroelektronik auf Implantaten ist ein Schritt in eine neue Ära der Frakturnachbehandlung [8]. Durch kabellose, unblutige Messung der Last, welche auf ein internes Fixateursystem einwirkt, kann ein Rückschluss auf den Verlauf der knöchernen Frakturheilung gezogen werden. Ein weiterentwickeltes kleines portables Lesegerät wird komfortabel ein kontinuierliches Monitoring der Belastung in vivo ermöglichen.

Erste Ergebnisse bei Patienten mit Femurpseudarthrosen zeigen eine gute Korrelation zwischen der knöchernen Heilung und dem Verlauf der empfangenen Signale. Bei krankengymnastischen Übungen bzw. Belastung der betroffenen Extremität können sofort Spitzenbelastungen des Implantats aufgezeigt werden. Ziel ist eine telemetrisch kontrollierte Nachbehandlung von Osteosynthesen mit optimaler Ausnutzung der Belastungsmöglichkeit der Extremität, ohne eine Überlastung des Implantats zu provozieren. Zudem wird durch dieses System eine frühzeitige Erkennung von Problemen in der Frakturheilung ermöglicht werden, sodass entsprechend gegengesteuert werden kann. Nach Überzeugung der Autoren werden „intelligente“
Implantate für die Osteosynthese in der Zukunft wichtige Werkzeuge des Unfallchirurgen/Orthopäden sein.

\section{Fazit}

Insbesondere bei problematischen Frakturverläufen mit mehrfachen Revisionen bzw. mehreren Verfahrenswechsel hat sich das intelligente Implantat als sicheres Verfahren erwiesen, um eine knöcherne Heilung zu erreichen. Es konnten alle mittels intelligentem Implantat operierten Heilverläufe abgeschlossen werden.

\section{Korrespondenzadresse}

Dr. M. Faschingbauer

Abteilung für Unfall- und Wiederherstellungschirurgie, BG-Unfallkrankenhaus Hamburg, Bergedorfer Straße 10, 21033 Hamburg m.faschingbauer@buk-hamburg.de

Interessenkonflikt. Der korrespondierende Autor gibt an, dass kein Interessenkonflikt besteht.

\section{Literatur}

1. Burny F, Donkerwolcke M, Bourgois R et al. (1984) Twenty years experience in fracture healing measurement with strain gauges. Orthopaedics 7: 1823-1826

2. Claes $L$ (1991) Die Messung der Knochenheilung bei Fixateur-externe-Osteosynthesen mit dem Fraktometer FM 100. Chirurg 62: 354-355

3. Richardson JB, Cunningham JL, Goodship AE et al. (1994) Measuring stiffness can define healing of tibial fractures. J Bone Joint Surg Br 76B: 389-394

4. Rohlmann A, Bergmann G, Graichen F et al. (1995) In vivo measurement of implant loads in a patient with a fractured vertebral body. Eur Spine J 4: 347353

5. Schneider E, Michel MC, Genge M et al. (2001) Loads acting in an intramedullary nail during fracture healing in the human femur. J Biomech 34: 849857

6. Seide K, Weinrich N, Wenzl ME et al. (2004) Threedimensional load measurements in an external fixator. J Biomech 37: 1361-1369

7. Weinrich N, Seide K, Jürgens C et al. (2004) Telemetric system for load measurements on internal fixator systems. Beiträge zur 38. Jahrestagung der DGBMT (BMT 2004). Biomed Techn 49: 448-449

8. Wolter D, Seide K (1997) Das intelligente Implantat. In: Schneider E (Hrsg) Biomechanik des menschlichen Bewegungsapparats. Hefte Unfallchir 261: 236-240 